\title{
Leaving colorectal polyps in place can be achieved with high accuracy using blue light imaging (BLI)
}

United European Gastroenterology Journal 2018, Vol. 6(7) 1099-1105 (C) Author(s) 2018 Reprints and permissions: sagepub.co.uk/journalsPermissions.nav DOI: $10.1177 / 2050640618769731$ journals.sagepub.com/home/ueg @SAGE

\author{
Helmut Neumann ${ }^{1}$, Helmut Neumann sen. ${ }^{2}$, Michael Vieth ${ }^{3}$, \\ Raf Bisschops $^{4}$, Florian Thieringer ${ }^{1}$, Khan F. Rahman ${ }^{1}$, \\ Thomas Gamstätter ${ }^{1}$, Gian Eugenio Tontini ${ }^{5}$ and Peter R. Galle ${ }^{1}$
}

\begin{abstract}
Objectives: A negative predictive value of more than $90 \%$ is proposed by the American Society of Gastrointestinal Endoscopy Preservation and Incorporation of Valuable Endoscopic Innovations (PIVI) statement for a new technology in order to leave distal diminutive colorectal polyps in place without resection. To our knowledge, no prior prospective study has yet evaluated the feasibility of the most recently introduced blue light imaging (BLI) system for real-time endoscopic prediction of polyp histology for the specific endpoint of leaving hyperplastic polyps in place.

Aims: Prospective assessment of real-time prediction of colorectal polyps by using BLI.

Material and methods: In total, 177 consecutive patients undergoing screening or surveillance colonoscopy were included. Colorectal polyps were evaluated in real-time by using high-definition endoscopy and the BLI technology without optical magnification. Before resection, the endoscopist described each polyp according to size, shape and surface characteristics (pit and vascular pattern, colour and depression), and histology was predicted with a level of confidence (high or low).

Results: Histology was predicted with high confidence in $92.5 \%$ of polyps. Sensitivity of BLI for prediction of adenomatous histology was $92.68 \%$, with a specificity and accuracy of 94.87 and $93.75 \%$, respectively. Following the recommendation of the PIVI statement, positive and negative predictive values were calculated with values of 95 and $92.5 \%$, respectively. Prediction of surveillance based on both US and European guidelines was correctly predicted in $91 \%$ of patients.

Conclusion: The most recently introduced $\mathrm{BLI}$ technology is accurate enough to leave distal colorectal polyps in place without resection. BLI also allowed for assignment of postpolypectomy surveillance intervals. This approach therefore has the potential to reduce costs and risks associated with the redundant removal of diminutive colorectal polyps.
\end{abstract}

\section{Keywords}

$\mathrm{BLI}$, endoscopy, PIVI, polyp, advanced imaging, $\mathrm{LCl}$

Received: 16 January 2018; accepted: 18 March 2018

\section{Introduction}

The treatment of distal diminutive polyps less than $5 \mathrm{~mm}$ in size remains a global challenge in gastrointestinal endoscopy. Although endoscopic therapy is generally not difficult, the costs for the redundant removal of those polyps are enormous given the fact that most of the diminutive polyps have a low prevalence of advanced histologic features, with less than $1 \%$ of them showing a cancerous phenotype. ${ }^{1}$ Therefore, the American Society of Gastrointestinal Endoscopy (ASGE) has proposed the Preservation and Incorporation of Valuable Endoscopic Innovations

\footnotetext{
${ }^{1}$ First Medical Department, Interdisciplinary Endoscopy, University Medical Center Mainz, Mainz, Germany

${ }^{2}$ Internistische Schwerpunktpraxis Bad Salzuflen, Germany

${ }^{3}$ Institute of Pathology, Klinikum Bayreuth, Germany

${ }^{4}$ Department of Gastroenterology, University Hospital Gasthuisberg, Leuven, Belgium

${ }^{5}$ Gastroenterology and Endoscopy Unit, Fondazione IRCCS Ca'Granda Ospedale Maggiore Policlinico, Milan, Italy

Corresponding author:

Helmut Neumann, First Medical Department, Interdisciplinary Endoscopy, University Medical Center Mainz, Langenbeckstr. 1, 55131 Mainz, Germany. Email: helmut.neumann@unimedizin-mainz.de
} 
(PIVI) thresholds for real-time endoscopic assessment of the histology of diminutive colorectal polyps, proposing a negative predictive value of greater than $90 \%$ for a technology to guide the decision of leaving suspected rectosigmoid hyperplastic polyps less than $5 \mathrm{~mm}$ in diameter in place without resection. ${ }^{1,2}$ In addition, the ASGE proposed that polyps less than $5 \mathrm{~mm}$ or less can be resected and discarded without pathological assessment if a new technology provides $90 \%$ or greater agreement in assignment of the correct postpolypectomy surveillance interval. The implementation of the resect and discard strategy would result in a potential upfront saving, which was calculated as more than one billion dollars in the USA. ${ }^{3}$

Meanwhile, the PIVI recommendations are recognised as one important parameter highlighting the effectiveness of advanced endoscopic imaging modalities. To date, various studies have shown the potential of different imaging techniques, including Narrow Band Imaging (NBI), Flexible spectral Imaging Colour Enhancement (FICE) and i-scan for the prediction of colorectal polyp histology. ${ }^{4-6}$

Most recently, a new imaging system was introduced to flexible endoscopy system methods that are implemented in the distal tip of a high-definition endoscope.

Here, we prospectively assessed for the first time the potential of the most recently introduced BLI-system for real-time prediction of distal colorectal polyp histology according to the recommendations of the ASGE PIVI statement for colorectal polyps.

\section{Material and methods}

\section{Study design, setting and patient recruitment}

Consecutive patients undergoing screening or surveillance colonoscopy were prospectively included. The study was approved by the ethics committee (Code 103_14) and was conducted according to the ethical guidelines of the 1975 Declaration of Helsinki, as reflected in a prior approval by the institution's human research committee. Written, informed consent was obtained from each patient included in the study. Patients with a history of inflammatory bowel disease, poor bowel preparation, coagulative disorders or polyposis syndromes were not included.

\section{The BLI system}

For the purpose of this study, the newly introduced VP-7000 video processor was used in combination with the latest high-definition video colonoscopes (Fujifilm, Tokyo, Japan). Images were displayed on $27^{\prime \prime}$ Ultra LED (light-emitting diode) monitors (Radiance, NDS, USA) providing a typical luminance of $900 \mathrm{~cd} / \mathrm{m}^{2}$ at $6500^{\circ} \mathrm{K}$ colour temperature. The endoscopy system is equipped with Complementary metal-oxide-semiconductor (CMOS) and 4-LED multilight technology consisting of four independently acting LEDs. The specific light spectrum consists of blueviolet, blue, green and red. Enhanced visualization of haemoglobin, and thus blood vessels, is generated by the high peak intensity of short-wavelength light. The BLI system produces a peak of high intensity at $410 \mathrm{~nm}$, which resembles the absorption maximum of haemoglobin. The modified visible light spectrum results in an increased visualization of the mucosal surface and vascular pattern morphology as compared to standard white light imaging.

\section{Endoscopic and histologic assessment of colorectal polyps}

Prior to the study, the endoscopist underwent a 1-hour online teaching lesson on pit pattern analysis of colorectal polyps. Afterwards, a training set containing 20 images with known histology was reviewed to standardize image interpretation. Endoscopic imaging was performed using a standard technique after standard bowel preparation with either sodium picosulfate or polyethylene glycol solution in split dose regime before the examination. Patients receiving conscious sedation were monitored according to the guidelines of the German society. ${ }^{7}$ On withdrawal from the cecum, all parts of the colon were carefully evaluated for lesions. Once a polyp was detected, it was photographed in white light mode, followed by BLI. The location, size (as compared to an open biopsy forceps or snare) and Paris classification of the polyp were described. ${ }^{8}$ Using the BLI system, surface characteristics were described including pit pattern, vascular pattern morphology and colour. Afterwards, the polyp was removed either with a biopsy forceps for lesions less than $3 \mathrm{~mm}$ in size or in conventional snare technique. After resection, the lesion was put in buffered formalin and send for subsequent histopathological analysis, which was performed by an expert pathologist. The pathologist was blinded to the real-time prediction of colorectal polyp histology. Finally, endoscopic prediction of colorectal polyp histology and histology were compared.

\section{Statistics}

All statistical analysis was done using PASW Statistics 18 (SPSS, Inc., Chicago, USA). A two-sided $P$ value $<0.05$ was considered to be significant. For comparisons of proportions we used Fisher's exact test. The median in this study is presented for non-normally distributed variables, while the mean is presented for normally distributed variables. The range 
Table 1. Patient demographics.

\begin{tabular}{lr}
\hline Gender, $n(\%)$ & \\
Male & $69(39)$ \\
Female & $108(61)$ \\
Age, years & \\
Mean \pm SD & $60 \pm 16.9$ \\
Median (range) & $59.88(21-89)$ \\
\hline
\end{tabular}

provided indicated the minimum and maximum values. To assess if BLI is reliable for assessment of postpolypectomy surveillance intervals, endoscopic predictions of polyp histology were compared to those recommended by pathological assessment for both European and US guidelines. ${ }^{9,10}$

For the purpose of this study, the confidence level was set to $95 \%$ with a confidence interval (CI) of $5 \%$ and a population proportion of $50 \%$. With a chosen population size of 100 this resulted in a calculated sample size of 80 polyps.

\section{Results}

\section{Study cohort}

In total, 177 consecutive patients (mean age 60 years; 108 female) undergoing screening or surveillance colonoscopy were included (Table 1). In 56 patients, a total of 87 colorectal polyps were detected (Figure 1). Overall, histology was available for 80 polyps thereby complying with the sample size calculation. Pathology revealed adenomatous phenotypes and hyperplastic phenotypes in 41 and 39 cases, respectively. In total, five sessile serrated adenomas were detected among three patients (Figure 1).

\section{Diagnostic performance of $B L I$}

Colorectal polyps were evaluated in real-time by using high-definition endoscopy and the BLI technology without optical magnification. Before resection, the endoscopist described each polyp according to size, shape and surface characteristics (pit and vascular pattern, colour and depression), and histology was predicted with a level of confidence (high or low).

The histology was predicted with high confidence in $92.5 \%$ of polyps with an overall accuracy of $93.75 \%$ (Figures 2 and 3). Of the 41 adenomas, 3 were incorrectly predicted to be hyperplastic lesions by BLI, while 2 of the 39 hyperplastic polyps were misdiagnosed as adenomas. All sessil serrated adenoma (SSA)s included in the study were correctly predicted as adenomatous lesions by BLI. Therefore, prediction of colorectal polyp histology with BLI showed a sensitivity of

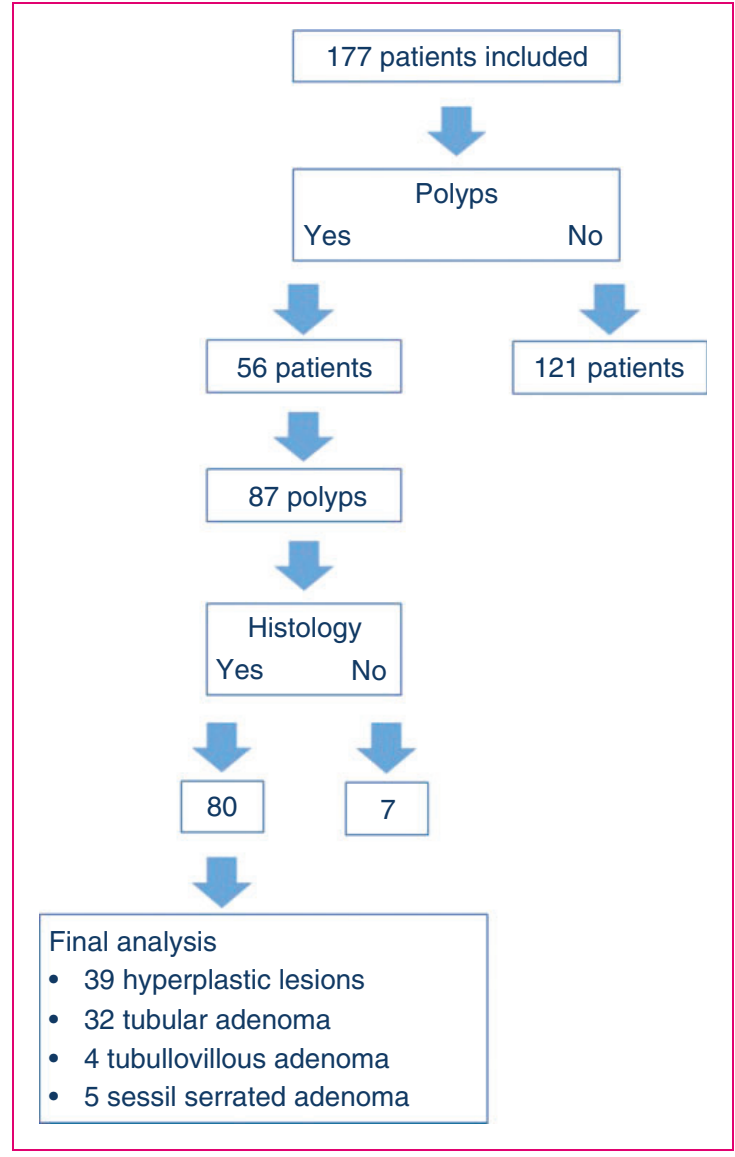

Figure 1. Study flow diagram.

$92.68 \%(95 \%$ CI $0.79-0.98)$ with a specificity of $94.87 \%$ (95\% CI 0.81-0.99). Following the recommendation of the PIVI statement, positive and negative predictive values were calculated with a value of $95 \%$ (95\% CI $0.82-0.99)$ and $92.5 \%$ (95\% CI $0.79-0.98)$, respectively (Table 2 ).

\section{Prediction of surveillance intervals with BLI}

In order to assess if BLI is reliable for assessment of postpolypectomy surveillance intervals, endoscopic predictions of polyp histology were compared to those recommended by pathological assessment for both European and US guidelines. ${ }^{9,10}$

Taking prediction of all colorectal polyps in this study together, surveillance based on both US and European guidelines was correctly predicted in 49 out of 54 patients $(91 \%$; data of two patients were not evaluable). In the five cases where surveillance intervals differed, according to the European guidelines BLI predicted shorter surveillance intervals in two patients (5 years instead of 10 years) and longer surveillance intervals in three patients (10 years instead of 5 years) as compared to standard histopathology. According to 


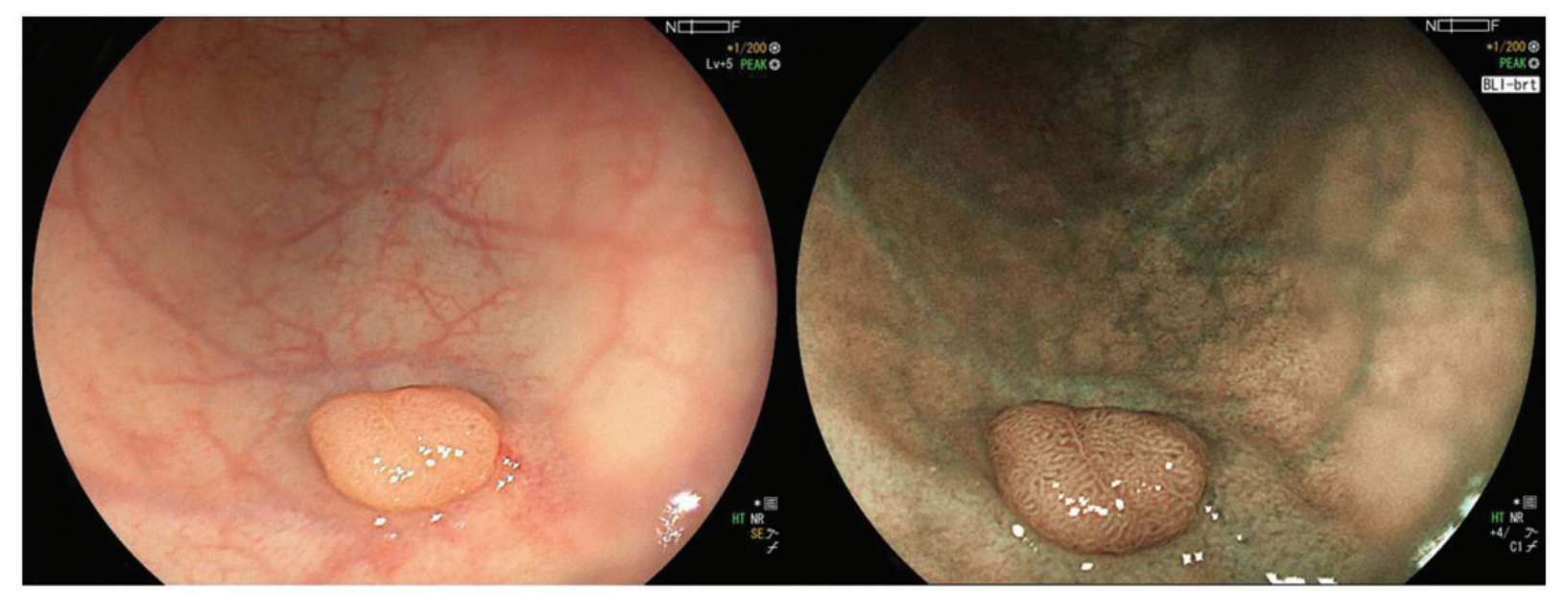

Figure 2. Tubular adenoma imaged with high-definition white light endoscopy (left) and blue light imaging (right). Blue light imaging allows for an adequate characterization of surface and vascular pattern details.

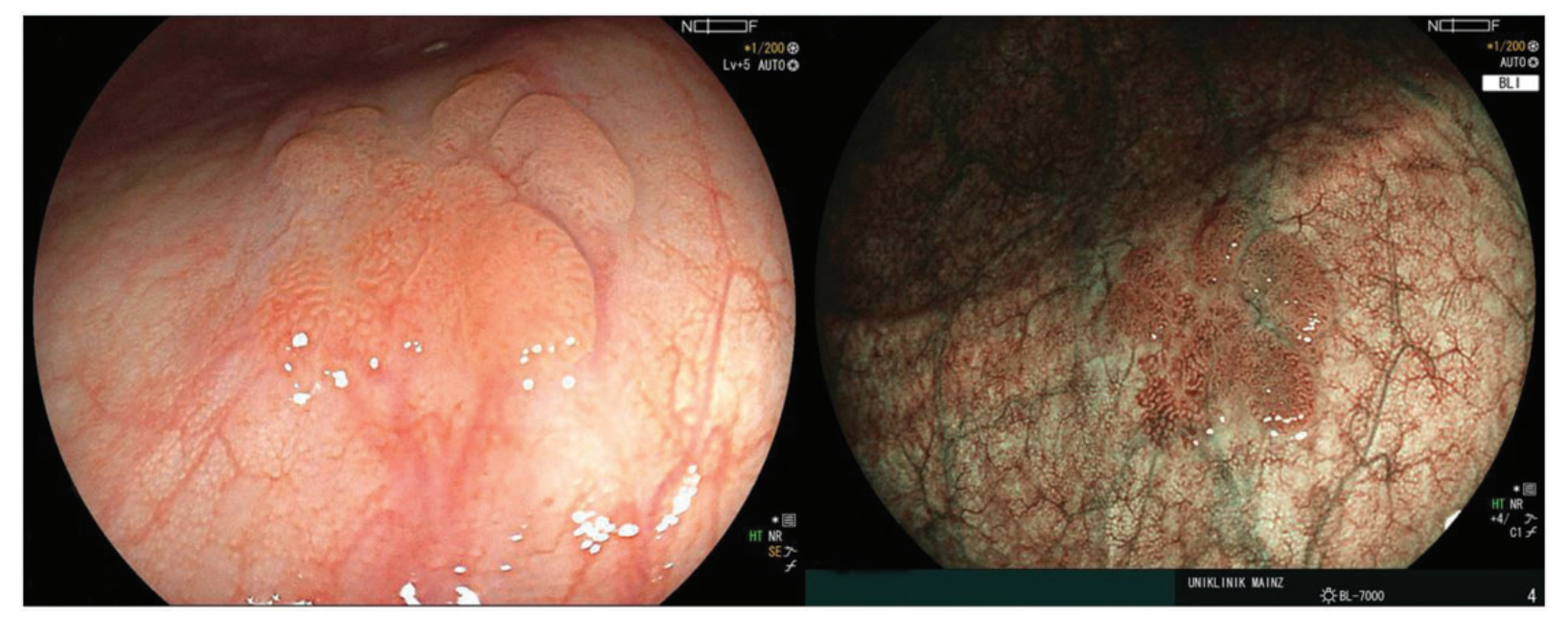

Figure 3. Sessile serrated adenoma highlighted with high-definition white light endoscopy (left) and blue light imaging (right). Blue light imaging enables clear demarcation of the non-polypoid lesion.

the US guidelines, no differences were observed between prediction based on BLI and standard histopathology (Table 3).

\section{Discussion}

Here, we have shown for the first time that the most recently introduced BLI technique, which is based on tissue illumination with LEDs, allows for accurate prediction of colorectal polyp histology according to the recommendations of the PIVI statement for colorectal polyps as proposed by the ASGE. Of note, the study has shown that BLI allows for both guidance for the decision to leave suspected hyperplastic polyps in place without resection and for the assignment of postpolypectomy surveillance intervals.

Colorectal polyps are a common condition regularly found during screening colonoscopy and the adenoma is considered as the precursor lesion of colorectal cancer. ${ }^{11}$ Therefore, adequate detection and resection of polyps is of utmost importance for an effective screening procedure. However, only up to $3 \%$ of polyps less than $5 \mathrm{~mm}$ in size show an adenomatous histology with a wide range reported in the literature between $0.15-3.4 \%{ }^{12,13}$ Most polyps, especially those located in the rectosigmoid area, demonstrate a 
Table 2. Diagnostic performance parameters of blue light imaging for prediction of polyp histology.

\begin{tabular}{lllll}
\hline Accuracy & $\begin{array}{l}\text { Sensitivity } \\
(95 \% \mathrm{Cl})\end{array}$ & $\begin{array}{l}\text { Specificity } \\
(95 \% \mathrm{Cl})\end{array}$ & $\begin{array}{l}\text { PPV } \\
(95 \% \mathrm{Cl})\end{array}$ & $\begin{array}{l}\text { NPV } \\
(95 \% \mathrm{Cl})\end{array}$ \\
\hline $93.75 \%$ & $92.68 \%$ & $94.87 \%$ & $95 \%$ & $92.5 \%$ \\
& $(0.79-0.98)$ & $(0.81-0.99)$ & $(0.82-0.99)$ & $(0.79-0.98)$
\end{tabular}

$\mathrm{Cl}$ : confidence interval; NPV: negative predictive value; PPV: positive predictive value.

Table 3. Prediction of surveillance intervals based on blue light imaging and standard histopathology according to US and European guidelines. ${ }^{9,10}$

\begin{tabular}{lrrrrr}
\hline & $\begin{array}{c}\text { Prediction based on } \\
\text { pathology (in years) }\end{array}$ & & \multicolumn{2}{c}{$\begin{array}{l}\text { Prediction based on } \\
\text { endoscopy (in years) }\end{array}$} \\
\cline { 2 - 3 } \cline { 5 - 6 } \cline { 5 - 6 } & US & European & & US & European \\
\hline Patient 1 & 10 & 10 & & $5-10$ & 5 \\
Patient 2 & 10 & 10 & & $5-10$ & 5 \\
Patient 3 & $5-10$ & 5 & & 10 & 10 \\
Patient 4 & $5-10$ & 5 & & 10 & 10 \\
Patient 5 & $5-10$ & 5 & & 10 & 10 \\
\hline
\end{tabular}

hyperplastic phenotype. In addition, most polyps in the rectosigmoid area are diminutive lesions measuring less than $5 \mathrm{~mm}$ in size. Therefore, accurate in vivo histology of colorectal polyps seems to be an appropriate strategy for the identification of polyps that to not possess a risk of developing colorectal cancer in order to reduce costs and the potential risks associated with their redundant removal. ${ }^{14}$ The PIVI recommendations have been well established to demonstrate the effectiveness for in vivo diagnosis of diminutive rectosigmoid polyps with a new endoscopic imaging technique.

In the present study, we have evaluated for the first time the potential of the most recently introduced BLI system for prediction of colorectal polyp histology and the assignment of postpolypectomy surveillance intervals. BLI is based on tissue illumination with LEDs and utilizes computer-based light transmission instead of filter technologies. Traditionally, dye-less chromoendoscopy techniques are based on optical filters (e.g. NBI, compound band imaging (CBI), i-scan optical enhancement (OE) and variable intelligence staining technology (VIST)) or digital postprocessing (e.g. FICE, SPIES and i-scan). ${ }^{15,16}$ Optical filters typically narrow the red light, therefore enhancing tissue illumination with blue light resulting in improved contrast of the surface pattern and vascular pattern morphology. In contrast, digital postprocessing uses transcoding software algorithms, which predominantly result in an improved visualization of the surface pattern morphology.
One recent randomized clinical trial evaluated whether optical diagnosis with NBI of diminutive colorectal polyps meets clinical practice standards and reduces the need for histopathology. ${ }^{17}$ In this trial, 558 subjects were enrolled and randomly assigned to near-focus NBI and standard view optical diagnosis. Endoscopists were significantly more likely to make a high-confidence optical diagnosis with near-focus $(85 \%)$ than standard $(73 \%)$ view. High-confidence diagnoses had 96 and $92 \%$ negative predictive value, respectively. Moreover, optical versus histopathological diagnosis showed excellent agreement between the surveillance intervals. Similar results have been reported by various other groups over the past few years. ${ }^{18,19}$

Similar to optical chromoendoscopy, recent data have shown the effectiveness of digital chromoendoscopy techniques for the evaluation of colorectal polyps, highlighting the effectiveness of digital chromoendoscopy for distinguishing neoplastic and nonneoplastic colorectal lesions. ${ }^{20}$

However, critical results regarding the effectiveness of dye-less chromoendoscopy techniques and their potential for in vivo diagnosis of colorectal polyps have also been published. Basford et al. compared high-definition white light endoscopy and i-scan for the assessment of small colon polyps and measured their performance against the PIVI thresholds for the assessment of diminutive colon polyps. ${ }^{21}$ A total of 209 polyps in 84 patients were included. There were no significant differences between white light endoscopy and i-scan in the characterization of small polyps. The negative predictive value for adenomatous histology of diminutive rectosigmoid polyps was $100 \%$ with both imaging techniques studied. UK and US polyp surveillance intervals were predicted with $95 \%$ accuracy with white light and $97 \%$ accuracy with i-scan. In addition, one recent prospective, randomized, multicentre trial reported that the levels of accuracy for real-time prediction of polyp histology did not differ between NBI and high-definition white light for optical diagnosis. ${ }^{22}$

Of note, the term 'BLI' is used twice in endoscopy. In our study, we have studied for the first time the potential of the blue light imaging system, which is based on LEDs, for the prediction of polyp histology. However, the term BLI is also used for blue laser imaging, an advanced endoscopic imaging technique that is not available in Europe or the Americas. Blue laser imaging (Lasereo, Fujifilm, Tokyo, Japan) utilizes two different laser systems, one with a wavelength of $450 \mathrm{~nm}$ passing through phosphor to irradiate white light and one with a wavelength of $410 \mathrm{~nm}$ for imaging of the vascular pattern morphology. ${ }^{23}$ Limited data on the technique are available, although preliminary data have already shown its potential for the diagnosis of colorectal polyps. ${ }^{24}$ 
Potential limitations of our study need to be acknowledged. First, the study was conducted as a single-centre study. However, no data are yet available on the potential of the BLI technique for the diagnosis of colorectal polyps. Therefore, our study now allows multicentre trials to be set up with adequate sample size calculation. Second, endoscopies were performed by an expert endoscopist. Accordingly, the results may be not the same for community physicians. Further data are therefore required addressing the learning curve and the applicability of the new technique during routine clinical practice. However, recent data on the realtime characterization of diminutive colorectal polyp histology using NBI has already shown that, with standardized training programmes, gastroenterologists without prior expertise in NBI were able to meet the negative predictive value and surveillance interval thresholds set forth by the ASGE. ${ }^{25}$ Therefore, one can potentially anticipate similar outcomes for the BLI technique. Last, the number of serrated lesions included was considerably low. Although BLI predicted adenomatous phenotypes correctly in all of those cases, the low number of serrated lesions included does not allow for a valid conclusion regarding the applicability of BLI for this specific type of lesion. Therefore, future research should specifically focus on the practicability of using BLI for the diagnosis of serrated lesions.

In summary, we have shown for the first time that the new LED-based imaging technique BLI is accurate enough to predict histology. This concept therefore has the potential to leave distal diminutive colorectal hyperplastic polyps in place without resection or for them to be resected without subsequent histopathological analysis. In addition, we have shown that the BLI technology allows for the assignment of postpolypectomy surveillance intervals according to the recommendations of the ASGE PIVI statement. Although our results are promising, we recommend the confirmation of our results in a multicentre study, including lessexperienced physicians at different training levels. National and international guidelines should also address the legal aspects of these new diagnostic possibilities in more detail.

\section{Declaration of conflicting interests}

The authors declared no potential conflicts of interest with respect to the research, authorship and/or publication of this article. Helmut Neumann and Raf Bisschops act as consultants for Fujifilm.

\section{Funding}

Endoscopy equipment was provided by Fujifilm Europe.

\section{Ethics approval}

The study was approved by the ethics committee (Code 103_14) and was conducted according to the ethical guidelines of the 1975 Declaration of Helsinki, as reflected in a prior approval by the institution's human research committee.

\section{Informed consent}

Written, informed consent was obtained from each patient included in the study.

\section{References}

1. Rex DK, Kahi C, O'Brien M, et al. The American Society for Gastrointestinal Endoscopy PIVI (Preservation and Incorporation of Valuable Endoscopic Innovations) on real-time endoscopic assessment of the histology of diminutive colorectal polyps. Gastrointest Endosc 2011; 73: 419-422.

2. ASGE Technology Committee, Abu Dayyeh BK, Thosani N, et al. ASGE Technology Committee systematic review and meta-analysis assessing the ASGE PIVI thresholds for adopting real-time endoscopic assessment of the histology of diminutive colorectal polyps. Gastrointest Endosc 2015; 81: 502.e1-502e16.

3. Kessler WR, Imperiale TF, Klein RW, et al. A quantitative assessment of the risks and cost savings of forgoing histologic examination of diminutive polyps. Endoscopy 2011; 43: 683-691.

4. Singh R, Jayanna M, Navadgi S, et al. Narrow-band imaging with dual focus magnification in differentiating colorectal neoplasia. Dig Endosc 2013; 25: 16-20.

5. Hoffman A, Loth L, Rey JW, et al. High definition plus colonoscopy combined with i-scan tone enhancement vs. high definition colonoscopy for colorectal neoplasia: A randomized trial. Dig Liver Dis 2014; 46: 991-996.

6. Pohl J, Nguyen-Tat M, Pech O, et al. Computed virtual chromoendoscopy for classification of small colorectal lesions: A prospective comparative study. Am J Gastroenterol 2008; 103: 562-569.

7. Riphaus A, Wehrmann T, Weber B, et al. S3-guidelinessedation in gastrointestinal endoscopy. Z Gastroenterol 2008; 46: 1298-1330.

8. Paris Workshop Participants. The Paris endoscopic classification of superficial neoplastic lesions: Esophagus, stomach, and colon: November 30 to December 1, 2002. Gastrointest Endosc 2003; 58: 3-43.

9. Valori R, Rey JF, Atkin WS, et al. European guidelines for quality assurance in colorectal cancer screening and diagnosis. First Edition-Quality assurance in endoscopy in colorectal cancer screening and diagnosis. Endoscopy 2012; 44: 88-105.

10. Lieberman DA, Rex DK, Winawer SJ, et al. Guidelines for colonoscopy surveillance after screening and polypectomy: A consensus update by the US Multi-Society Task Force on Colorectal Cancer. Gastroenterology 2012; 143: 844-857. 
11. Ishaq S, Siau K, Harrison E, et al. Technological advances for improving adenoma detection rates: The changing face of colonoscopy. Dig Liver Dis 2017; 49: 721-727.

12. Neumann $\mathrm{H}$ and Hassan C. Small and diminutive polyps: No cancer, no risk! Dig Liver Dis 2017; 49: 1-2.

13. Rex DK, Hassan C and Bourke MJ. The colonoscopist's guide to the vocabulary of colorectal neoplasia: Histology, morphology, and management. Gastrointest Endosc 2017; 86: 253-263.

14. Wanders LK, East JE, Uitentuis SE, et al. Diagnostic performance of narrowed spectrum endoscopy, autofluorescence imaging, and confocal laser endomicroscopy for optical diagnosis of colonic polyps: A meta-analysis. Lancet Oncol 2013; 14: 1337-1347.

15. Neumann H, Fujishiro M, Wilcox CM, et al. Present and future perspectives of virtual chromoendoscopy with i-scan and optical enhancement technology. Dig Endosc 2014; 26: 43-51.

16. Tontini GE and Neumann H. Endoscopic classification for colorectal tumors using narrow-band imaging. Dig Endosc 2016; 28: 537-538.

17. Kaltenbach T, Rastogi A, Rouse RV, et al. Real-time optical diagnosis for diminutive colorectal polyps using narrow-band imaging: the VALID randomised clinical trial. Gut 2015; 64: 1569-1577.

18. Paggi S, Rondonotti E, Amato A, et al. Narrow-band imaging in the prediction of surveillance intervals after polypectomy in community practice. Endoscopy 2015; 47: $808-814$.
19. Repici A, Hassan C, Radaelli F, et al. Accuracy of narrow-band imaging in predicting colonoscopy surveillance intervals and histology of distal diminutive polyps: Results from a multicenter, prospective trial. Gastrointest Endosc 2013; 78: 106-114.

20. Albrecht H, Nägel A, Tasdelen H, et al. Digital chromoendoscopy with i-Scan for in vivo prediction of advanced colorectal neoplasia: A multicenter study. J Clin Gastroenterol 2016; 50: 91-94.

21. Basford PJ, Longcroft-Wheaton G, Higgins B, et al. High-definition endoscopy with i-Scan for evaluation of small colon polyps: The HiSCOPE study. Gastrointest Endosc 2014; 79: 111-118.

22. Klare P, Haller B and Wormbt S. Narrow-band imaging vs. high definition white light for optical diagnosis of small colorectal polyps: A randomized multicenter trial. Endoscopy 2016; 48: 909-915.

23. Shimoda R, Sakata Y, Fujise T, et al. The adenoma miss rate of blue-laser imaging vs. white-light imaging during colonoscopy: A randomized tandem trial. Endoscopy 2017; 49: 186-190.

24. Nakano A, Hirooka Y and Yamamura T. Comparison of the diagnostic ability of blue laser imaging magnification versus pit pattern analysis for colorectal polyps. Endosc Int Open 2017; 5: 224-231.

25. Patel SG, Schoenfeld P, Kim HM, et al. Real-time characterization of diminutive colorectal polyp histology using narrow-band imaging: Implications for the resect and discard strategy. Gastroenterology 2016; 150: 406-418. 